\title{
Rejuvenation of Facial Skin by Transplantation of the Autologous Stromal Vascular Fraction Isolated Using a New Type of Clinical-Grade Collagenase
}

Jiaqi Qin

Shanghai 9th Peoples Hospital Affiliated to Shanghai Jiaotong University School of Medicine

Bin Fang

Shanghai 9th Peoples Hospital Affiliated to Shanghai Jiaotong University School of Medicine Jizhou He

Shanghai 9th Peoples Hospital Affiliated to Shanghai Jiaotong University School of Medicine

Chen Cheng

Shanghai 9th Peoples Hospital Affiliated to Shanghai Jiaotong University School of Medicine

Jia Zhou

Shanghai 9th Peoples Hospital Affiliated to Shanghai Jiaotong University School of Medicine

Shuangbai Zhou

Shanghai 9th Peoples Hospital Affiliated to Shanghai Jiaotong University School of Medicine

Rulin Huang

Shanghai 9th Peoples Hospital Affiliated to Shanghai Jiaotong University School of Medicine

\section{Poh-ching Tan}

Shanghai 9th Peoples Hospital Affiliated to Shanghai Jiaotong University School of Medicine

Tianyu Zhang

Xi'an Tieyi Binhe School

Taoran Jiang

Shanghai 9th Peoples Hospital Affiliated to Shanghai Jiaotong University School of Medicine

Wenhui Liu

First Affiliated Hospital of Zhengzhou University

Hairong Wang

Shanghai 9th Peoples Hospital Affiliated to Shanghai Jiaotong University School of Medicine Yun Xie ( $\boldsymbol{\sigma}$ drxieyun@shsmu.edu.cn)

Shanghai 9th Peoples Hospital Affiliated to Shanghai Jiaotong University School of Medicine https://orcid.org/0000-0002-4341-7426

Qingfeng Li

Shanghai 9th Peoples Hospital Affiliated to Shanghai Jiaotong University School of Medicine 
Keywords: stromal vascular fraction (SVF), collagenase, adipose tissue-derived mesenchymal stem cells (ASCs)

Posted Date: January 31st, 2022

DOI: https://doi.org/10.21203/rs.3.rs-1283507/v1

License: (c) (1) This work is licensed under a Creative Commons Attribution 4.0 International License. Read Full License 


\section{Abstract \\ OBJECTIVE}

The purpose of this study was to detect the yield, activity and safety of the stromal vascular fraction (SVF) isolated by a new clinical-grade collagenase with a specific process and to validate the efficacy of the method.

\section{MATERIALS AND METHODS}

For the in vitro study, adipose tissue specimens were obtained from women undergoing elective liposuction $(n=5)$. The SVF was isolated by new clinical-grade collagenase for the experimental group and research-grade collagenase NB4 for the control group. The surface markers of the SVFs isolated by the two collagenases were detected by flow cytometry. The yield, activity and morphology of the SVFs were compared, together with their proliferation, migration and differentiation. Furthermore, we conducted a clinical trial to evaluate the improvement of skin texture after transplantation of autologous SVF isolated by clinical-grade collagenase.

\section{RESULTS}

There were no statistically significant differences in the yield, viability or morphology of the SVFs isolated by the new collagenase and collagenase NB4. Adipose tissue-derived mesenchymal stem cells (ASCs) surface marker expression was consistent between the two products. The differentiation potential of the cells was not different, which was consistent with previously published results. The SVF isolated by the new collagenase had a higher proliferation and migration. After administration of autologous SVF isolated by the new collagenase, the VISIA score improved significantly with regard to wrinkle scores and texture scores.

\section{CONCLUSION}

We concluded that this new type of clinical-grade collagenase can replace current research-grade products without any negative effect on the yield or function of the SVF, which can be applied to antiaging and rejuvenation of facial skin.

\section{Introduction}

In recent years, stem cell therapy has rapidly evolved and is aimed towards an increasing number of diseases. Bone marrow mesenchymal stem cells (BM-MSCs) and adipose tissue-derived mesenchymal stem cells (ASCs) are currently the most widely used stem cells. There are 100-1000-fold more MSCs in 
adipose tissue than in bone marrow ${ }^{1}$, and it is less invasive and less painful to obtain adipose tissue, which has led to a focus on ASCs.

The stromal vascular fraction (SVF) is obtained from adipose tissue after collagenase digestion, filtration, and centrifugation. This fraction contains not only abundant adipose tissue-derived stem cells (ASCs) but also a heterogeneous cell population, including endothelial cells, adipose precursor cells, monocytes, and macrophages ${ }^{2}$, which expands the application of the SVF. Currently, the SVF has been used in the fields of plastic surgery, rheumatology, musculoskeletal conditions, chronic wounds, urogenital diseases, cardiovascular respiratory diseases, etc. ${ }^{2-7}$, which has led to major progress in its clinical use.

Nonetheless, standard isolation methods have not yet been established for obtaining clinical-grade cells ${ }^{8}$. Since Rodbell and Jones first developed a method for isolating SVF cells from adipose tissue in 1964, a variety of methods for isolating the SVF have been used ${ }^{9-11}$. There are two methods commonly used to obtain the SVF: enzymatic and mechanical. ${ }^{3}$ Tissue enzymatic digestion is now the most widely used method due to its high cellular yield ${ }^{12}$. However, most published protocols employ research-grade collagenase containing xenogeneic components, which may cause severe anaphylaxis, immune reactions, or infections in patients ${ }^{13}$. For some products, the enzymatic composition shows lot-to-lot variability that may affect reproducibility ${ }^{14}$.

In this study, the researchers sought to apply a new clinical-grade collagenase to digest adipose tissue and determine the yield, activity, safety and efficacy of the resulting SVF. This new type of clinical-grade collagenase mainly consists of aseptically filled, highly purified collagenase, which is approved only for the treatment of herniated discs with nucleus pulposus in China. A clinical trial was also conducted to determine the changes in skin texture following the application of this clinical-grade collagenase-digested SVF for facial grafting.

\section{Materials And Methods}

\section{Patients and samples}

Adipose tissue specimens were obtained from five healthy donors who underwent abdominal liposuction with informed consent from the Shanghai Ninth People's Hospital. This study was approved by the Institutional Ethics Committee of the Shanghai Ninth People's Hospital.

\section{The enzymatic products}

Here, we used a new type of clinical-grade collagenase (Shanghai Joy Biopharm Co., Ltd.) to digest adipose tissue for SVF as the experimental group. We thoroughly mixed 600 units of collagenase and 2 $\mathrm{ml}$ of saline solution to obtain the collagenase solution. Collagenase NB4 (Sigma) solution was used as the control.

\section{Preexperiment}


We performed preliminary experiments and found that collagenase concentrations of $45 \mathrm{U} / \mathrm{ml}$ for 45 minutes and $60 \mathrm{U} / \mathrm{ml}$ for 45 minutes were superior for obtaining the SVF (Supplementary Material 1).

\section{Assays for cell proliferation}

Cell proliferation was analysed by Cell Counting Kit-8 assays. SVFs treated with different concentrations of collagenase were seeded and cultured in 96-well microplates, and collagenase NB4 was used as a control. After treatment for 24, 48 and 72 hours, CCK-8 reagent was added to each well and cultured for 2 hours. The absorbance was analysed at $450 \mathrm{~nm}$ using a microplate reader, which displayed the proliferation of cells.

\section{Assays for cell migration}

In the cell scratch assay, SVFs were cultured on a six-well plate to the monolayer state, and a blank area was artificially created. After 12 and 24 hours, the migrated cells on the underside of the filters were counted under a microscope within at least five randomly chosen fields, and then, the wound closure rate was calculated.

\section{Isolation and culture of SVFs}

Fat was harvested from the abdominal tissue. The rinsed human fat was placed in a centrifuge tube. The prepared enzymatic products were added to digest the lipoaspirate at $37^{\circ} \mathrm{C}$ for 60 minutes. The incubated tube was centrifuged to remove the upper layer, and the lower layer was resuspended in saline solution. After filtering, we washed the filter residue by centrifugation, and the sunken cell mass was the SVF. Finally, the SVF was cultured and expanded to adipose stem cells (ASCs).

\section{Yield, viability and morphology of the SVF}

We counted the cells in the obtained SVF in an automatic cell counter to obtain the number of live cells. The sample was then assessed under an electron microscope for morphological observation.

\section{Immunophenotypic analysis of the SVF}

For flow cytometric analysis, the ASCs were incubated with antibodies to different CD molecules. Isotype control IgG was used to stain the cells as a control. The cells were subsequently washed with phosphatebuffered saline (PBS), fixed with $4 \%$ formaldehyde and analysed on a FACScan flow cytometer.

\section{Multipotential induction}

ASCs were cultured in adipogenic induction medium for the designated time and stained by Oil Red O. For osteogenic differentiation, cells were cultured in osteogenic induction medium for the designated time and stained with Alizarin red S. Chondrogenic differentiation was performed using the micromass culture technique and chondrogenic medium for up to 5 weeks, followed by staining with Alcian blue. 


\section{SVF acquisition and injection in patients}

All of our patients were females aged 30 to 56 years old. Twelve of them underwent the liposuction procedure from the abdomen and one from the double upper arms. The SVF was isolated by the new type of clinical-grade collagenase from the lipoaspirate, and then, we injected the SVF at an amount of $2.5 \times 10^{7}$ nucleated cells per side into the forehead, lower eyelid and crow's feet. The other side was used as the control. The patients were followed up for one year, and the VISIA score was used to assess facial improvement.

\section{Results}

1. The collagenase solution had a higher digestive efficiency, and digestion at an enzyme concentration of $45 \mathrm{U} / \mathrm{ml}$ for 45 minutes maximized cell proliferation and migration.

We conducted an experiment to demonstrate that adding saline solution in an equal volume of fat can improve the SVF digestion efficiency of collagenase. (Figure 1A) The proliferative capacity of cells was analysed by Cell Counting Kit-8 (CCK-8) assays. The SVF digested with an enzyme concentration of 45 $\mathrm{U} / \mathrm{ml}$ for 45 minutes had optimal proliferation. (Figure 1B) Next, we performed cell scratch experiments to measure the migration of the cells. The SVF digested with an enzyme concentration of $45 \mathrm{U} / \mathrm{ml}$ for 45 minutes exhibited the best migration. (Figure 1C)

2. There were no significant differences in the yield, viability or morphology of the SVFs isolated by the new collagenase and collagenase NB4.

To test the yield of the SVFs digested by the new collagenase and collagenase NB4, we counted the total cells harvested per $20 \mathrm{ml}$ of lipoaspirate, and the results were not significantly different. (Figure 2A) There was no obvious change in viability between the SVFs isolated by the new collagenase and collagenase NB4 after we calculated the live cells per HPF for several days. (Figure 2B) The morphology of the cells under electron microscopy also showed no major discrepancy. (Figure 2C)

3. ASC surface marker expression and the differentiation potential were consistent between the SVFs isolated by the new collagenase and collagenase NB4.

We cultured the isolated SVF in the proper medium until we obtained ASCs. According to flow cytometry analysis of ASC-related surface markers, we concluded that consistent surface markers, such as CD14 and CD44, were expressed on the ASCs isolated by the new collagenase and collagenase NB4. (Figure 3A) To confirm the capacity of the cultured ASCs to differentiate into multiple cell types, we assessed the adipogenic, osteogenic, and chondrogenic potential of the cells in vitro. We used Oil Red $O$ staining for lipid droplet formation, Alizarin red S staining for calcium deposit formation and Alcian blue staining for sulphated proteoglycan formation, and there were no significant differences among them. (Figure 3B) 
4. After administration of autologous SVF isolated by the new collagenase, the VISIA score for wrinkles and texture were improved.

We conducted a clinical trial with 13 patients. The SVF isolated by the new collagenase was injected into the forehead, lower eyelid and crow's feet of one side, and the other side was self-controlled. During a follow-up period of more than one year, we compared the forehead, lower eyelid and crow's feet for wrinkle (Figure 4A) and texture (Figure 4B) and scored them with VISIA. We observed significant improvement in skin wrinkles and texture on the side injected with the SVF obtained with the new collagenase treatment.

\section{Discussion}

The stromal vascular fraction (SVF) is a heterogeneous cell population that includes endothelial cells, adipose precursor cells, monocytes, macrophages, and T cells. The therapeutic potential of the SVF has been demonstrated in several experimental and practical applications. For instance, the SVF can provide a cellular and molecular microenvironment for ASCs; in wound healing and cardiovascular diseases, the SVF can enhance angiogenesis and neovascularization ${ }^{7}$; it can significantly improve the survival rate of fat grafts ${ }^{15-17}$ and promote vascularization and collagen remodelling in ageing skin ${ }^{18}$. SVF isolated by research-grade collagenase, which contains heterogeneous components, cannot be widely used because the safety is not acceptable. In the present study, we utilized a new type of clinical-grade collagenase to digest lipoaspirate and compared this SVF with that isolated by current research-grade collagenase.

The new type of clinical-grade collagenase is currently used clinically in orthopaedics for the treatment of herniated disc nucleus pulposus in China. We used this clinical-grade collagenase to digest lipoaspirate and compared the SVF with that obtained by conventional research-grade collagenase (collagenase NB4) for various properties. We first determined the digestion concentration of collagenase through a series of pre-experiments. At this concentration, the proliferation and migration of the cells were detected. The results showed that the cells isolated by $45 \mathrm{~min}$ of digestion with $45 \mathrm{U} / \mathrm{ml}$ collagenase solution had higher proliferation and migration. By counting the cells isolated and detecting the number of viable cells, we found no significant difference in the yield and cellular activity of the SVFs isolated by the two collagenases. The morphology of the SVFs was also generally consistent. ASCs were obtained by culturing the isolated SVF in medium for a period of time, and flow cytometry was performed. There were no significant differences in the immunological markers between the two groups. Subsequently, we did not find any significant differences in the differentiation potential of the SVFs. Through the above results, we concluded that the SVF obtained with the new collagenase was very similar in biological properties to the SVF obtained by digestion with research collagenase and that the new collagenase can replace the current research-grade collagenase.

We also conducted a clinical trial. We performed liposuction on 13 enrolled subjects, 12 from the abdomen and 1 from both upper arms. After treatment with the new type of clinical-grade collagenase, the SVF was injected into the forehead, lower eyelid and crow's feet on one side, and the other side served 
as its own control. During the follow-up period, we performed facial skin VISIA analysis on 13 subjects, focusing on wrinkle and texture scores. The results showed that injection of the SVF digested with this new collagenase improved the skin condition of the face.

Thus, our study establishes a new method for obtaining the SVF through the application of a new clinicalgrade collagenase, which solves the problem of heterogeneous composition encountered in the preparation of the SVF and demonstrates the positive effect of the SVF in facial rejuvenation, pushing the application of the SVF one step forward. However, the problems of complex SVF preparation methods and low yields still exist. There are already many companies working on closed, one-time SVF extraction devices, and some progress has been made. ${ }^{19}$ There is still a long way to go for the clinical application of SVF.

\section{Conclusion}

We concluded that this new type of clinical-grade collagenase can replace current research-grade products without any negative effect on the yield or function of the SVF, which can be applied to antiaging and rejuvenation of facial skin.

\section{Abbreviations}

SVF

Stromal vascular fraction

ASCs

Adipose tissue-derived mesenchymal stem cells (ASCs)

\section{Declarations}

\section{Ethics approval and consent to participate}

This study got the informed consent from the Shanghai Ninth People's Hospital and was approved by the Institutional Ethics Committee of the Shanghai Ninth People's Hospital. Our clinical trial registration has been completed on the ClinicalTrial.gov PRS. (NCT03189628)

\section{Consent for publication}

Not applicable.

\section{Availability of data and materials}

The datasets used and/or analysed during the current study are available from the corresponding author on reasonable request.

\section{Competing interests}


The authors declare that they have no competing interests.

\section{Funding}

This work was supported by the Two-hundred Talent (20191916) \Shanghai Municipal Key Clinical

Specialty (shslczdzk00901区and Fundamental research program funding of Ninth People's Hospital affiliated to Shanghai Jiao Tong university School of Medicine(JYZZ125).

\section{Authors' contributions}

JQ conducted the article writing. BF tested the number of living cells, sorted out the data and revised the article. JH did cell proliferation test. CC and SZ extracted SVF. JZ and RH are treatment researchers. PT took photos for skin examination during the follow-up of the case. TZ carried out SVF extraction method design and other related experiments. TJ screened the subjects. WL did the statistics. HW is a research nurse. QL is principal investigator. YX, a therapeutic researcher, conducted clinical trials and experimental design, improved SVF extraction methods and revised the paper.

All authors have read and approved the manuscript.

\section{Acknowledgements}

Not applicable.

\section{References}

1. Tsuji, W., Rubin, J. P. \& Marra, K. G. Adipose-derived stem cells: Implications in tissue regeneration. World J Stem Cells 6, 312-321, doi:10.4252/wjsc.v6.i3.312 (2014).

2. Han, S., Sun, H. M., Hwang, K. C. \& Kim, S. W. Adipose-Derived Stromal Vascular Fraction Cells: Update on Clinical Utility and Efficacy. Crit Rev Eukaryot Gene Expr 25, 145-152, doi:10.1615/critreveukaryotgeneexpr.2015013057 (2015).

3. Bora, P. \& Majumdar, A. S. Adipose tissue-derived stromal vascular fraction in regenerative medicine: a brief review on biology and translation. Stem Cell Res Ther 8, 145, doi:10.1186/s13287-017-0598-y (2017).

4. Jiang, S., Quan, Y., Wang, J., Cai, J. \& Lu, F. Fat Grafting for Facial Rejuvenation Using Stromal Vascular Fraction Gel Injection. Clin Plast Surg 47, 73-79, doi:10.1016/j.cps.2019.09.001 (2020).

5. Wolf, D. A. et al. Mesothelial Stem Cells and Stromal Vascular Fraction for Skin Rejuvenation. Facial Plast Surg Clin North Am 26, 513-532, doi:10.1016/j.fsc.2018.06.011 (2018).

6. Ramakrishnan, V. M. \& Boyd, N. L. The Adipose Stromal Vascular Fraction as a Complex Cellular Source for Tissue Engineering Applications. Tissue Eng Part B Rev 24, 289-299, doi:10.1089/ten.TEB.2017.0061 (2018). 
7. Andia, I., Maffulli, N. \& Burgos-Alonso, N. Stromal vascular fraction technologies and clinical applications. Expert Opin Biol Ther 19, 1289-1305, doi:10.1080/14712598.2019.1671970 (2019).

8. Zarei, F. \& Abbaszadeh, A. Application of Cell Therapy for Anti-Aging Facial Skin. Curr Stem Cell Res Ther 14, 244-248, doi:10.2174/1574888x13666181113113415 (2019).

9. Rodbell, M. METABOLISM OF ISOLATED FAT CELLS. I. EFFECTS OF HORMONES ON GLUCOSE METABOLISM AND LIPOLYSIS. J Biol Chem 239, 375-380 (1964).

10. Rodbell, M. The metabolism of isolated fat cells. IV. Regulation of release of protein by lipolytic hormones and insulin. J Biol Chem 241, 3909-3917 (1966).

11. Greenwood, M. R. \& Hirsch, J. Postnatal development of adipocyte cellularity in the normal rat. $J$ Lipid Res 15, 474-483 (1974).

12. Aronowitz, J. A., Lockhart, R. A. \& Hakakian, C. S. Mechanical versus enzymatic isolation of stromal vascular fraction cells from adipose tissue. Springerplus 4, 713, doi:10.1186/s40064-015-1509-2 (2015).

13. Carvalho, P. P., Gimble, J. M., Dias, I. R., Gomes, M. E. \& Reis, R. L. Xenofree enzymatic products for the isolation of human adipose-derived stromal/stem cells. Tissue Eng Part C Methods 19, 473-478, doi:10.1089/ten.TEC.2012.0465 (2013).

14. McCarthy, R. C., Breite, A. G., Green, M. L. \& Dwulet, F. E. Tissue dissociation enzymes for isolating human islets for transplantation: factors to consider in setting enzyme acceptance criteria. Transplantation 91, 137-145, doi:10.1097/TP.0b013e3181ffff7d (2011).

15. Matsumoto, D. et al. Cell-assisted lipotransfer: supportive use of human adipose-derived cells for soft tissue augmentation with lipoinjection. Tissue Eng 12, 3375-3382, doi:10.1089/ten.2006.12.3375 (2006).

16. Yoshimura, K. et al. Cell-assisted lipotransfer for cosmetic breast augmentation: supportive use of adipose-derived stem/stromal cells. Aesthetic Plast Surg 32, 48-55; discussion 56-47, doi:10.1007/s00266-007-9019-4 (2008).

17. Tanikawa, D. Y. S., Aguena, M., Bueno, D. F., Passos-Bueno, M. R. \& Alonso, N. Fat grafts supplemented with adipose-derived stromal cells in the rehabilitation of patients with craniofacial microsomia. Plast Reconstr Surg 132, 141-152, doi:10.1097/PRS.0b013e3182910a82 (2013).

18. Charles-de-Sá, L. et al. Antiaging treatment of the facial skin by fat graft and adipose-derived stem cells. Plast Reconstr Surg 135, 999-1009, doi:10.1097/prs.0000000000001123 (2015).

19. Brown, J. C. et al. Isolation of Adipose-Derived Stromal Vascular Fraction Cells Using a Novel Pointof-Care Device: Cell Characterization and Review of the Literature. Tissue Eng Part C Methods 23, 125-135, doi:10.1089/ten.TEC.2016.0377 (2017).

\section{Figures}




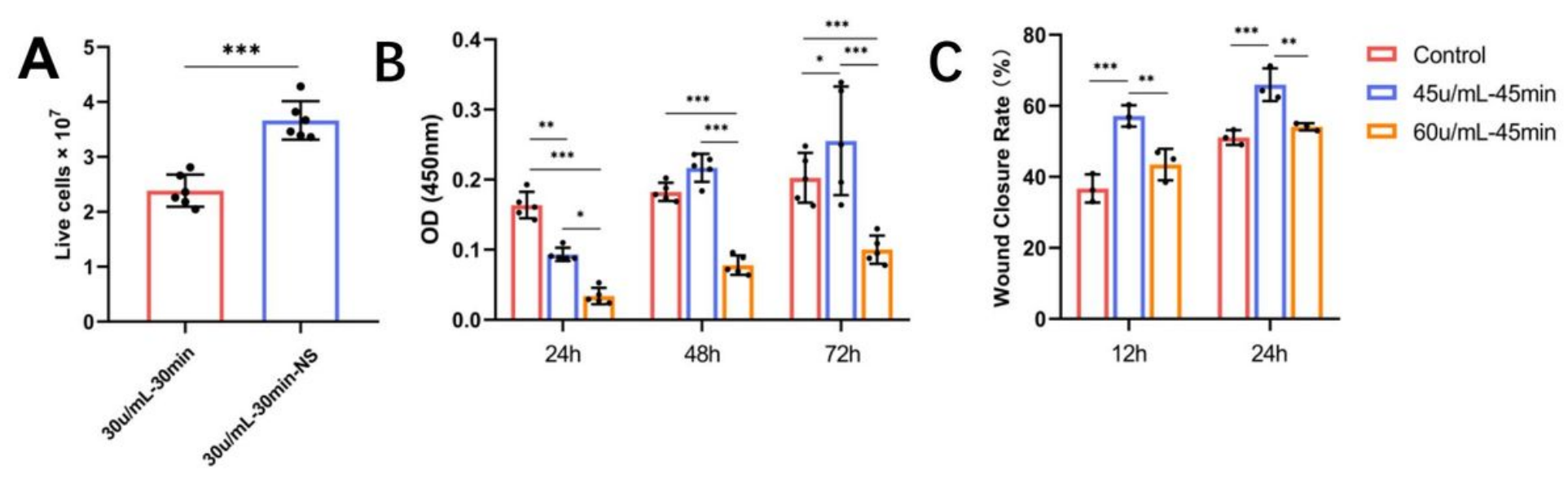

Figure 1

The effect of new collagenase concentration on cell properties. (A) The live cells in the SVF digested by the new collagenase solution with/without saline solution. (B) The absorbance of the SVFs isolated by different concentrations of the new collagenase and collagenase NB4. (C) The wound closure rate in the scratch assay produced by the SVFs isolated by different concentrations of the new collagenase and collagenase NB4.

A

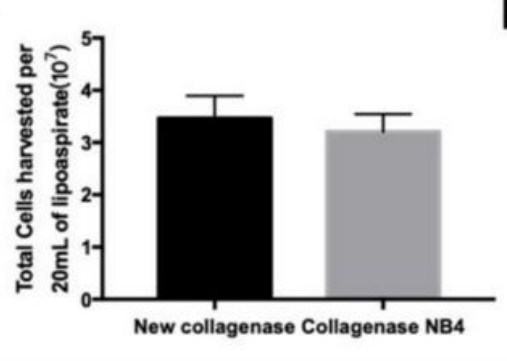

B

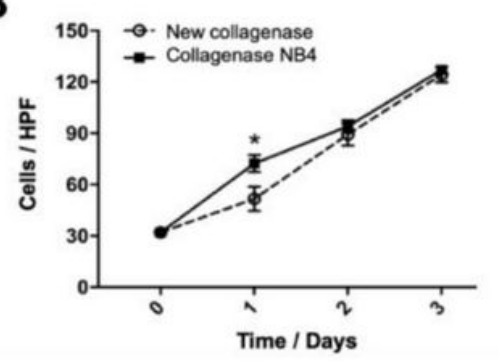

C

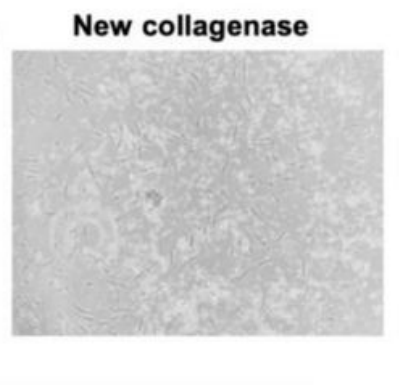

Collagenase NB4

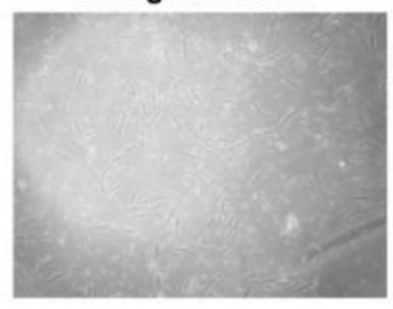

Figure 2

The effect of two enzymes on cellular characteristics. (A) The total SVFs harvested per $20 \mathrm{ml}$ of lipoaspirate isolated by the new collagenase and collagenase NB4 were calculated. (B) The number of surviving cells per HPF. (C) Representative images of cells isolated by the new collagenase and collagenase NB4. 

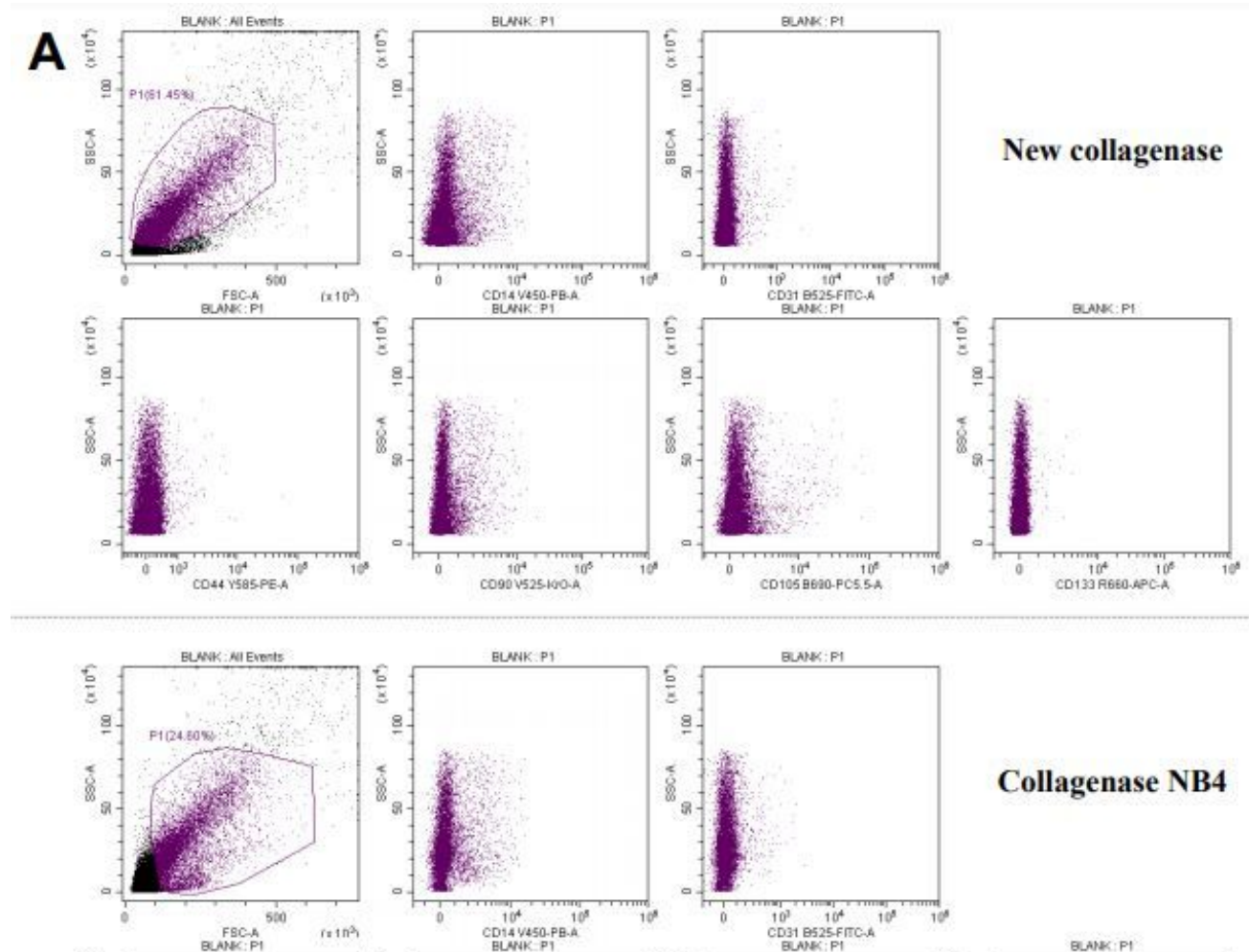

\section{Collagenase NB4}
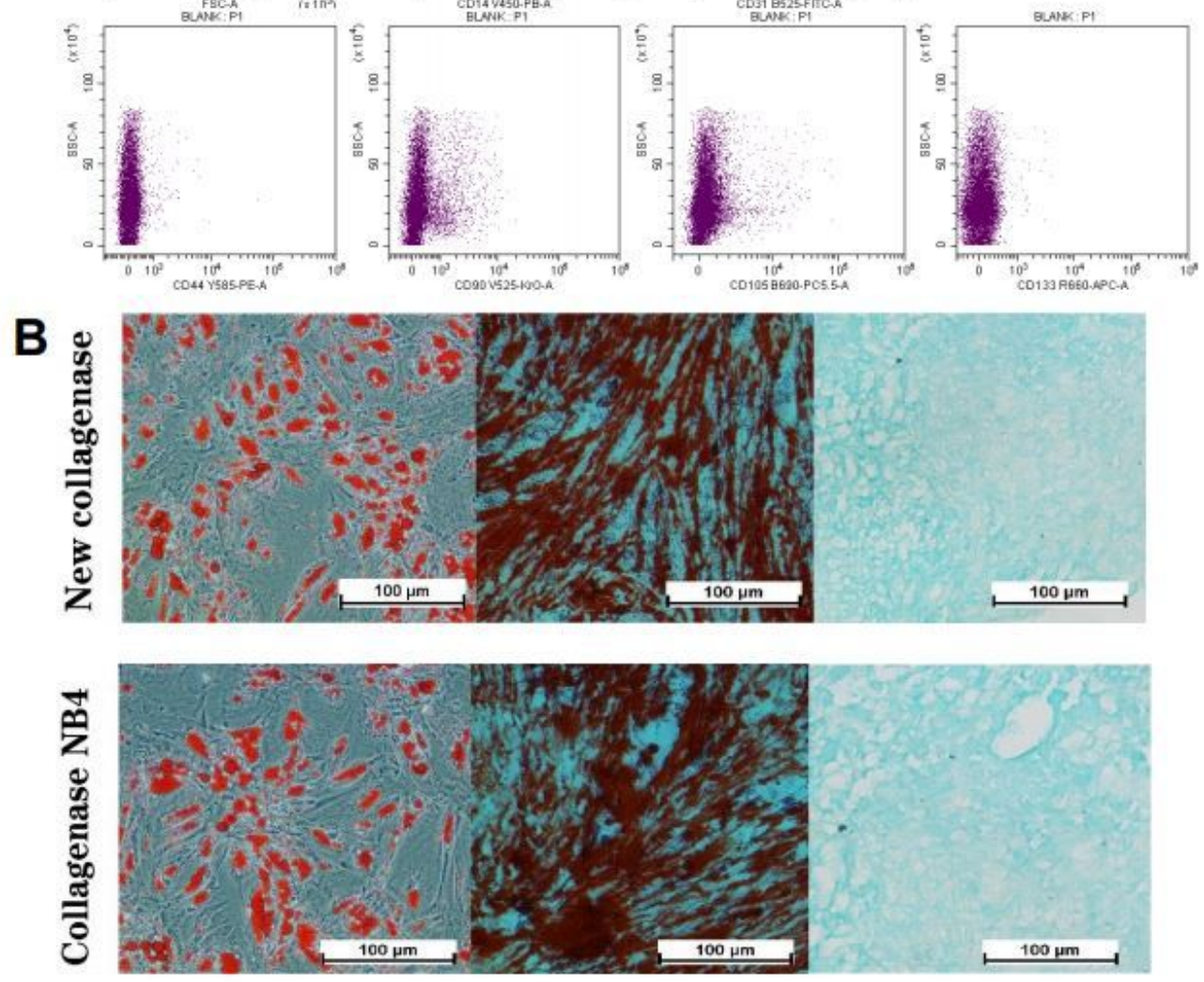

\section{Figure 3}

Flow cytometry and cell differentiation. (A) Immunophenotypic analysis of the SVFs isolated by the new collagenase and collagenase NB4 was performed by flow cytometry. (B) The SVFs isolated by the new collagenase and collagenase NB4 can differentiate to adipogenic, osteogenic and chondrogenic lineages in vitro, as demonstrated by positive Oil Red $\mathrm{O}$ staining, Alizarin red $\mathrm{S}$ staining and Alcian blue staining. Scale bar:100 $\mu \mathrm{m}$. 

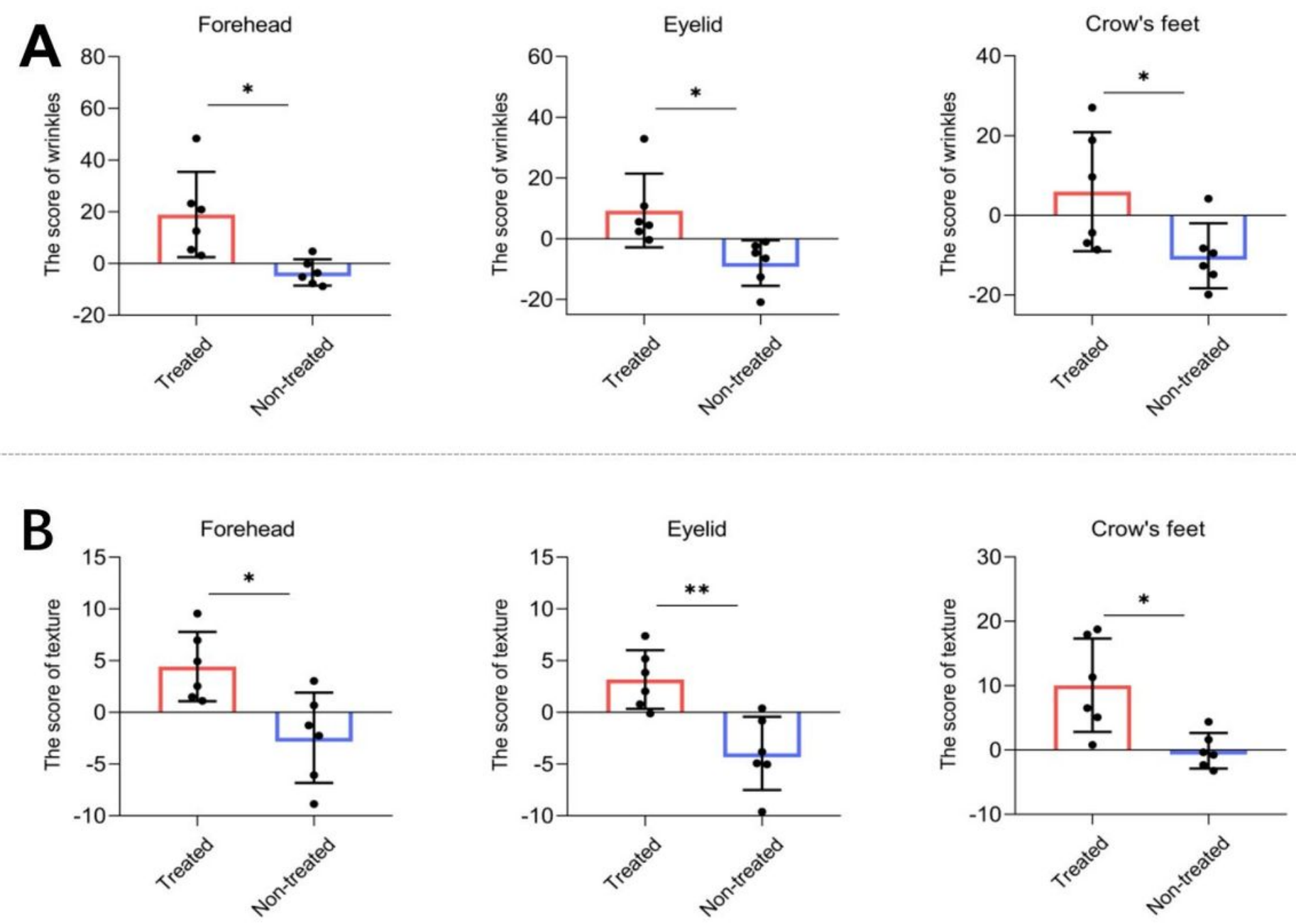

Figure 4

Facial improvement analysis. (A) The VISIA wrinkle scores for forehead, lower eyelid and crow's feet on the treatment side and the control side with SVF. (B) The VISIA texture scores for forehead, lower eyelid and crow's feet on the treatment side and the control side with SVF.

\section{Supplementary Files}

This is a list of supplementary files associated with this preprint. Click to download.

- Supplementarymetarial1.pdf 